\title{
Persistence and Lifelong Fidelity of Phase Singularities in Optical Random Waves
}

\author{
L. De Angelis, ${ }^{1,2}$ F. Alpeggiani, ${ }^{1,2}$ A. Di Falco, ${ }^{3}$ and L. Kuipers ${ }^{1,2, *}$ \\ ${ }^{1}$ Kavli Institute of Nanoscience, Delft University of Technology, 2600 GA Delft, The Netherlands \\ ${ }^{2}$ Center for Nanophotonics, AMOLF, Science Park 104, 1098 XG Amsterdam, The Netherlands \\ ${ }^{3}$ SUPA, School of Physics and Astronomy, University of St Andrews, North Haugh, St Andrews KY16 9SS, United Kingdom
}

(Received 25 July 2017; published 16 November 2017)

\begin{abstract}
Phase singularities are locations where light is twisted like a corkscrew, with positive or negative topological charge depending on the twisting direction. Among the multitude of singularities arising in random wave fields, some can be found at the same location, but only when they exhibit opposite topological charge, which results in their mutual annihilation. New pairs can be created as well. With nearfield experiments supported by theory and numerical simulations, we study the persistence and pairing statistics of phase singularities in random optical fields as a function of the excitation wavelength. We demonstrate how such entities can encrypt fundamental properties of the random fields in which they arise.
\end{abstract}

DOI: 10.1103/PhysRevLett.119.203903

A wide variety of physical systems exhibit vortices, locations around which an observable rotates while being undetermined in the middle [1-9]. It is especially fascinating when the properties and evolution of such singular entities can comprehensively describe complex phenomena, such as the Kosterlitz-Thouless transition [10]. But vortices are not a peculiarity of superconductors: light's phase twists around optical vortices, where it is singular [11]. A multitude of these phase singularities arises in random optical fields, one half twisting in opposite direction to the other, so that they can respectively approach to an arbitrarily small distance [12-16]. It is by letting them move that one can observe the creation and/or annihilation of such pairs [17-22].

With near-field experiments, we track phase singularities in a random optical field from birth to death. We map the singularities' trajectories at varying excitation wavelength, and quantitatively determine properties such as their persistence in the field and the correspondence between creation and annihilation partner of a singularity, known as lifelong fidelity [23]. We observe two populations of singularities, neatly differentiated by their typical persistence in the varying wave field: short-lived pairs that are predominantly faithful to their creation partner, and a more promiscuous population.

To generate optical random waves we couple monochromatic light $\left(\lambda_{0} \sim 1550 \mathrm{~nm}\right)$ into a photonic crystal chaotic cavity realized in a silicon-on-insulator platform [Fig. 1(a)] [24]. We map the optical field inside the cavity with near-field microscopy, resolving amplitude, phase, and polarization of such an in-plane complex electric field $\left(E_{x}, E_{y}\right)$ [25-27]. Figure 1 presents a typical example of our measurements of amplitude and phase of $E_{x}$. The optical field inside the cavity can be thought of as a random superposition of light waves [28] with transverse electric (TE) polarization [15]. Only the behavior of $E_{x}$ is presented here, without loss of generality, as it is representative of the behavior of all in-plane field components [15].

Figure 1(b) represents a full-size measurement: a square map $17 \times 17 \mu \mathrm{m}$ with a pixel size of about $17 \mathrm{~nm}$. In this map we distinguish a multitude of dark and bright spots, the results of destructive and constructive interference. Figures 1(c)-1(f) are $2 \times 2 \mu \mathrm{m}$ zoomed-in images of the full measurements taken at different wavelengths of the input light. Here, we can observe how a small change of the wavelength $(\delta=0.02 \mathrm{~nm})$ hardly changes the spatial pattern of the amplitude. Figures $1(\mathrm{~g})-1(\mathrm{j})$ depict the measured phase and reveal the phase singularities which take place at every zero in the amplitude (gray circles). We pinpoint the position of these singularities with deep subwavelength resolution and simultaneously determine their topological charge, which is always observed to be +1 (light circles) or -1 (dark circles), corresponding to a $\pm 2 \pi$ change of the phase around the singular point [29].

In Figs. 1(g)-1(j) we observe that the singularities move as a function of wavelength. More eye-catching than their tiny movements are the annihilation and creation events of pairs of singularities, which we can both observe between Figs. 1(h) and 1(i), highlighted by black circles. Indeed, singularities can be created and annihilated, but only in processes that conserve the total topological charge of the system, i.e., in pairs of opposite topological charge. Thus, as we tune the wavelength, singularities exhibit a transitory persistence in the random field over the span of a finite wavelength shift $\Delta \lambda$ between their creation and annihilation. In Fig. 2 we present a $3 \mathrm{D}$ representation of the trajectories followed in space and wavelength by a small subset of all the singularities that we measure. Among all the trajectories, the red ones represent lifelong faithful singularities: special cases where the singularities have the same partner for both creation and annihilation. The green trajectories are unfaithful singularities. 


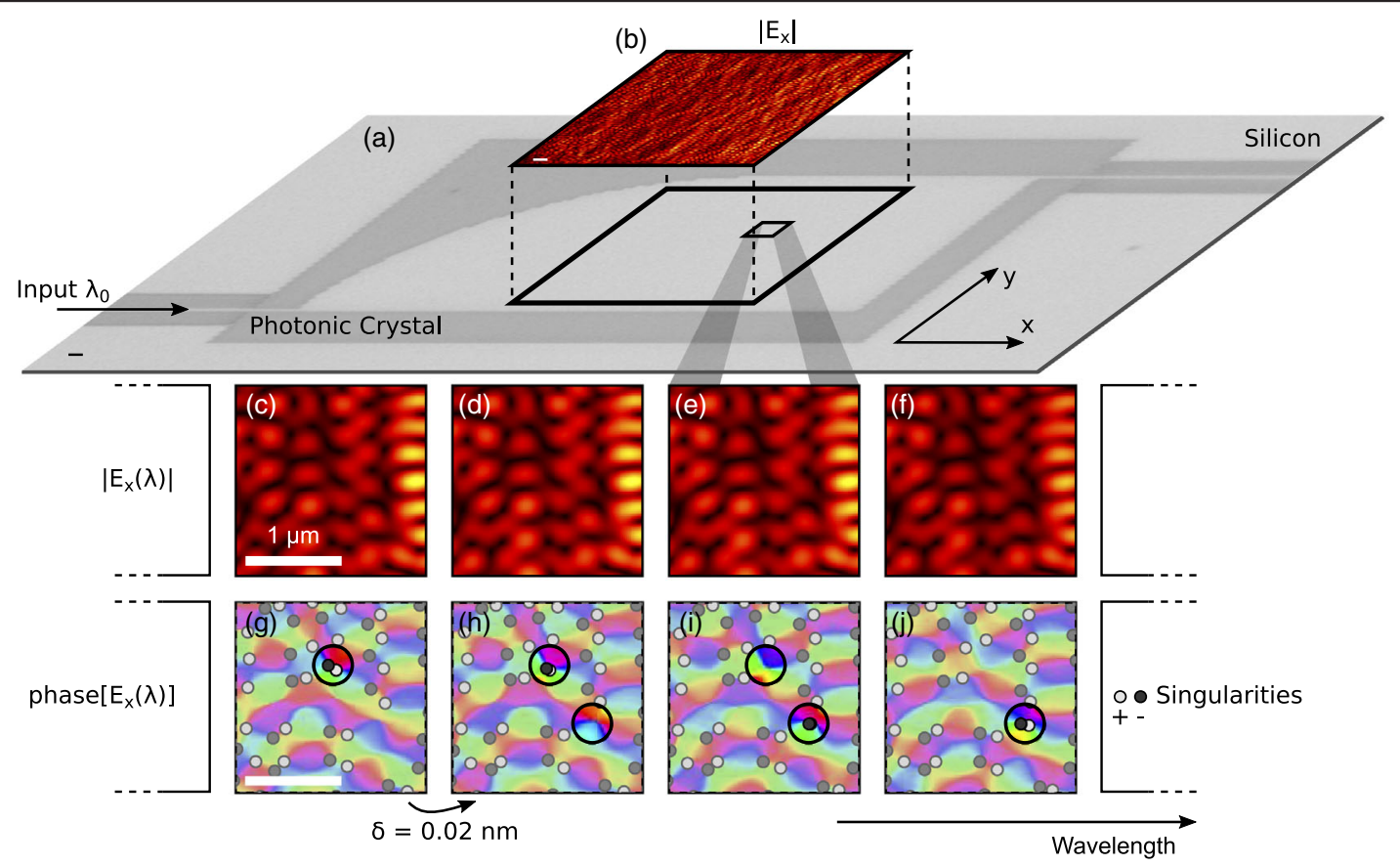

FIG. 1. Near-field measurements of the optical field in the chaotic cavity. (a) Optical micrograph of the chaotic cavity; the dark area is a photonic crystal that confines light inside the cavity. (b) A $17 \times 17 \mu$ m near-field map of the amplitude of the $x$ component of the electric field in the cavity. (c)-(f) Zoomed-in image of panel (b) for different input wavelengths $\lambda$. (g)-(j) False-color map of the measured phase for different input wavelengths $\lambda$; in such maps we pinpoint phase singularities with positive (dark spots) or negative (light spots) topological charge. The black circles highlight annihilation and creation events.

Figure 3(a) presents the number $\mathcal{N}$ of singularities as a function of their persistence $\Delta \lambda$ in the measured field. The main plot illustrates the results obtained from a data set in which we tuned the wavelength with a step $\delta=0.02 \mathrm{~nm}$ for a total range $\mathcal{L}=1.2 \mathrm{~nm}$. In this plot we clearly distinguish two persistence regimes separated by a cutoff wavelength shift $\lambda^{*} \approx 0.15 \mathrm{~nm}$. In the region $\Delta \lambda>\lambda^{*}$ the number of singularities exponentially decays against their

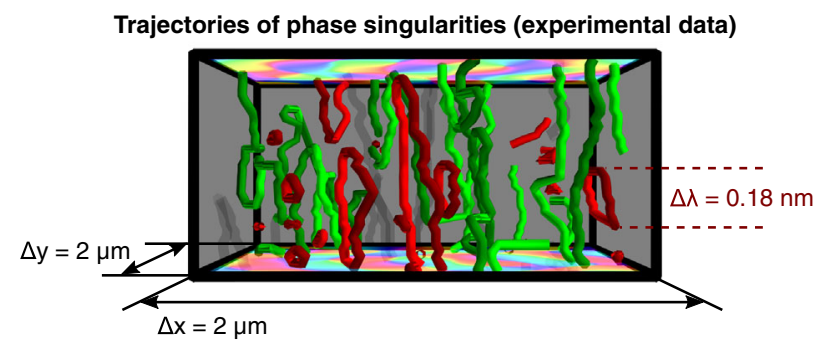

FIG. 2. A 3D representation of the trajectories of singularities that propagate through a $2 \mu \mathrm{m} \times 2 \mu \mathrm{m} \times 0.64 \mathrm{~nm}$ observation volume. The red and green trajectories are of faithful and unfaithful singularities, respectively. In both cases, bright and dark colors differentiate oppositely charged singularities. Please note that the parts of the trajectories that continue outside of the observation volume are not shown. The semitransparent trajectories are of singularities that propagate outside of the total wavelength range; these are not taken into account in our statistics. persistence in the field, with a characteristic persistence $\lambda_{d}=0.6 \mathrm{~nm}$. Such exponential behavior is even more clear from the data displayed in the inset, representative of a measurement in which the total range of the wavelength sweep is $\mathcal{L}=8 \mathrm{~nm}(\delta=0.1 \mathrm{~nm})$. Please note that the finite size of the wavelength scans has non-negligible effects on the measured statistics, mainly for the longest trajectories, thus slightly distorting the exponential behavior. This is because singularities that are created and/or annihilated outside the measured wavelength range need to be excluded from the persistence statistics. We estimate the fraction of such singularities to be of the order of $\Delta \lambda / \mathcal{L}$, resulting in a correction factor for $\mathcal{N}(\Delta \lambda)$ proportional to $(1-\Delta \lambda / \mathcal{L})$.

The exponential behavior that we observe could be interpreted in a straightforward way as the result of a memoryless Poisson process. However, we discover a physics richer than that. From the main plot of Fig. 3(a) we notice that $\mathcal{N}(\Delta \lambda)$ is not purely exponential: it clearly deviates from such a distribution for $\Delta \lambda$ smaller than $\lambda^{*}$. This spectral region contains an excess of singularities compared to what the asymptotic exponential distribution would predict. Their characteristic persistence in the field is much smaller than $\lambda_{d}$. In fact, by fitting $\mathcal{N}(\Delta \lambda)$ with a biexponential distribution, we can estimate the characteristic persistence for such a short-living population to be approximately $0.03 \mathrm{~nm}$. Note that the cutoff wavelength shift $\lambda^{*}$ does not depend on the absolute starting wavelength, as the trajectories of the singularities in excess are 


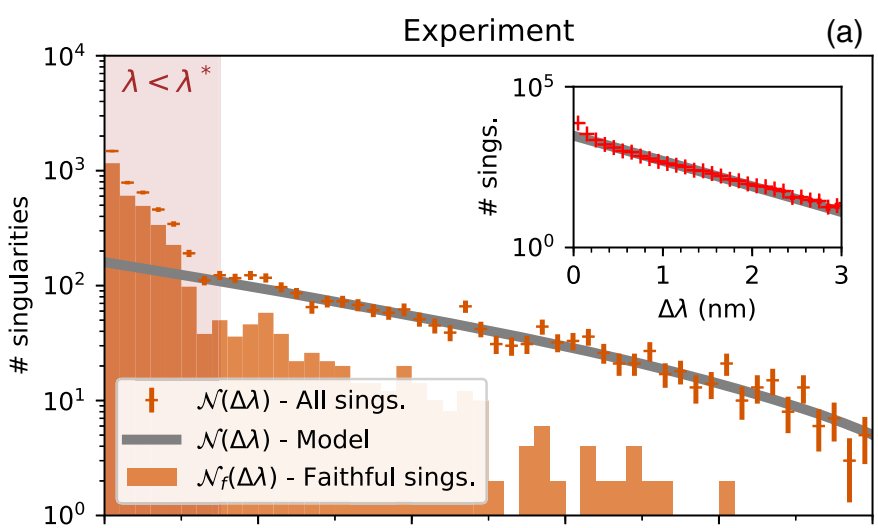

(a)

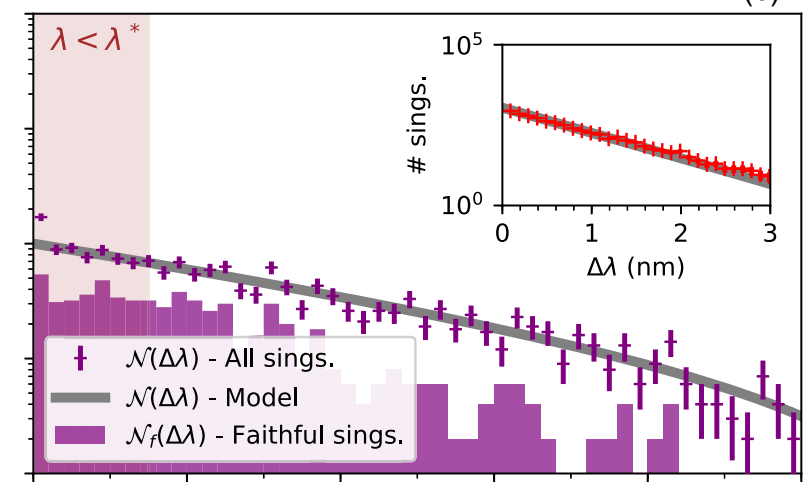

(c)

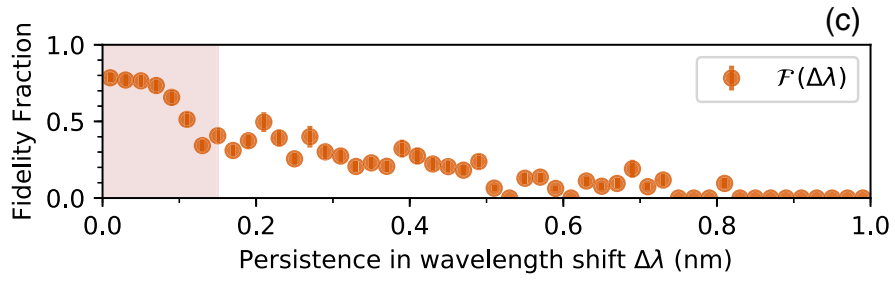

FEM simuation

(b)

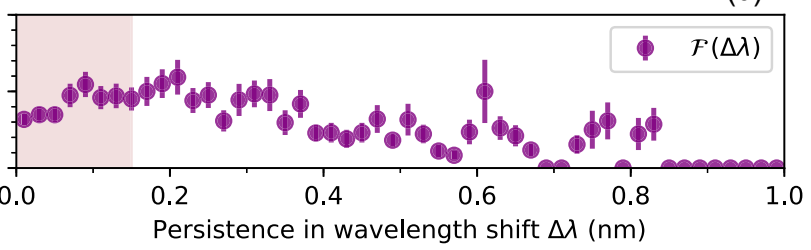

FIG. 3. Overview of the statistics for the persistence and fidelity of singularities in random waves, in experiment [(a),(c)] and finiteelement method (FEM) simulation [(b),(d)]. In the upper panels the dashes indicate the number of singularities $\mathcal{N}$ which persisted in the field for a given wavelength shift $\Delta \lambda$. The main figures refer to experiment or simulation where the wavelength was swept over a range $\mathcal{L}=1.2 \mathrm{~nm}$ with a step $\delta=0.02 \mathrm{~nm}$, whereas the insets show the results of wider scans $(\mathcal{L}=8 \mathrm{~nm}, \delta=0.1 \mathrm{~nm})$. The gray lines are representative of the prediction for the persistence histogram given by our model. In the same panels, the boxes are still a persistence histogram, but in which only the $\mathcal{N}_{f}$ singularities that were faithful to each other are taken into account. In the lower panels we report the fidelity fraction $\mathcal{F}$ of phase singularities, again as a function of their persistence in the field $\left[\mathcal{F}(\Delta \lambda)=\mathcal{N}{ }_{f}(\Delta \lambda) / \mathcal{N}(\Delta \lambda)\right]$.

uniformly distributed along the measured wavelength range.

Interestingly, when considering only the faithful singularities (red trajectories in Fig. 2), a different behavior is observed than for the full ensemble of all singularities. It is clear from Fig. 3(a) that in the region where deviation from a single exponential takes place $\left(\Delta \lambda<\lambda^{*}\right)$, we have an over-representation of faithful singularities (yellow boxes). This is reflected explicitly in the fidelity fraction $\mathcal{F}(\Delta \lambda)=$ $\mathcal{N}_{f}(\Delta \lambda) / \mathcal{N}(\Delta \lambda)$ represented in Fig. 3(c): while a majority of the short-living singularities are faithful to each other, the opposite is true for long-living ones.

The origin of the cutoff $\lambda^{*}$, which discriminates the population of faithful and short-living singularities from that of unfaithful and long-living ones, must be sought in the evolution properties of the field. Figure 4 displays the correlation coefficient of the considered experimental field at wavelengths $\lambda_{1}$ and $\lambda_{2}$ (upper left part),

$$
\rho\left(\lambda_{1}, \lambda_{2}\right)=\frac{\left|\int d \mathbf{r} \tilde{E}_{x}^{*}\left(\lambda_{1}\right) \tilde{E}_{x}\left(\lambda_{2}\right)\right|}{\sqrt{\int d \mathbf{r}\left|\tilde{E}_{x}\left(\lambda_{1}\right)\right|^{2} \int d \mathbf{r}\left|\tilde{E}_{x}\left(\lambda_{2}\right)\right|^{2}}},
$$

where $\tilde{E}_{x}=E_{x}-\left\langle E_{x}\right\rangle$. It is interesting to note that the correlation coefficient $\rho\left(\lambda_{1}, \lambda_{2}\right)$ decays over a finite wavelength shift $\lambda_{c} \approx \lambda^{*}$. Such a close relation between $\lambda_{c}$ and $\lambda^{*}$ may suggest that those singularities which spend their entire existence in the region of spectral correlation of the field exhibit persistence and pairing properties that are different from those of singularities overliving this region.

Although seemingly intuitive, this simple interpretation cannot be the whole story. We can firmly state this, having developed a model that creates a field with correlation properties analogous to those of our measured field, which in fact does not contain any excess of faithful singularities for small persistences.

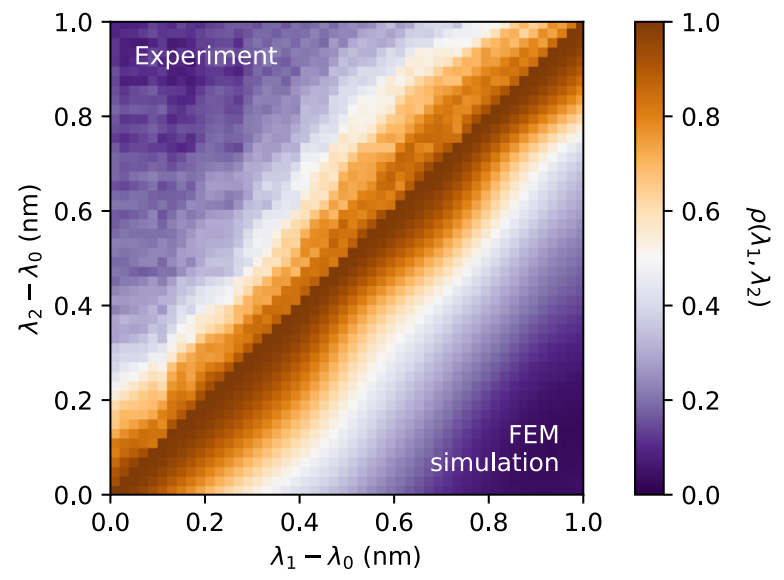

FIG. 4. Correlation coefficient $\rho\left(\lambda_{1}, \lambda_{2}\right)$ of the measured (upper left) and FEM-simulated (lower right) optical random field, at wavelengths $\lambda_{1}$ and $\lambda_{2}\left(\lambda_{0}=1550 \mathrm{~nm}\right)$. 
We model the frequency-dependent field as a superposition of the cavity eigenstates $\mathcal{E}_{x}^{j}$, centered at frequencies $\tilde{\omega}_{j}=\omega_{j}+i \gamma_{j}[28]$,

$$
E_{x}(\mathbf{r}, \omega)=\sum_{j} \frac{\alpha_{j} \mathcal{E}_{x}^{j}\left(\mathbf{r}, \omega_{j}\right)}{\omega-\left(\omega_{j}+i \gamma_{j}\right)} .
$$

Based on the size of our cavity, we can estimate the average spectral separation between two consecutive eigenstates $\Delta=\left\langle\omega_{j+1}-\omega_{j}\right\rangle_{j}$ to be approximately $0.08 \mathrm{~nm}$ [28]. In the simplest model field, we assume the eigenstates to be equidistant in frequency (spacing $\Delta$ ), with a constant width $\gamma$ and a unitary weight $\left(\alpha_{j}=1\right)$. We set $\gamma=0.16 \mathrm{~nm}$, equal to the average loss rate of our system, which we determine from a finite-difference time domain simulation of the entire three-dimensional chaotic cavity. Finally, following Berry's hypothesis [30], we consider every eigenstate to be a random superposition of monochromatic plane waves,

$$
\mathcal{E}_{x}^{j}\left(\mathbf{r}, \omega_{j}\right)=\sum_{|\mathbf{k}|=n \omega_{j} / c} a_{x, \mathbf{k}} \exp \left(i \mathbf{k} \cdot \mathbf{r}+i \delta_{\mathbf{k}}\right),
$$

where $\delta_{\mathbf{k}}$ is a random variable uniformly distributed in $[0,2 \pi]$ and $a_{x, \mathbf{k}} \propto|\mathbf{k} \times \hat{x}|$ is the polarization coefficient for the TE mode [15]. With these assumptions, we construct the wavelength-dependent field of Eq. (2) and determine the statistics of its singularities. We find that the persistence of singularities is exponentially distributed, with a characteristic decay rate that depends on the ratio between the eigenstates' width and spacing $\gamma / \Delta$. For our estimated parameters $(\gamma / \Delta \approx 2)$ this results in $\lambda_{d}^{\text {th }}=0.6 \mathrm{~nm}$. The persistence calculated with our model is presented in Fig. 3(a) (gray line). In the region $\Delta \lambda>\lambda^{*}$ we obtain perfect matching between experiment and theory.

These theoretical results are confirmed by a 2D FEM simulation. The FEM simulation produces a direct expression of $\mathbf{E}(\mathbf{r}, \omega)$, free of the previous model assumptions. The statistics for the singularities obtained from these simulations are reported in Figs. 3(b), 3(d). Comparing the simulated persistence histogram to our model calculation we notice perfect agreement at every wavelength shift. No deviation from a single-exponential distribution in the region $\Delta \lambda<\lambda^{*}$ is present, and in the same region the fidelity fraction is lower than experimentally observed [Figs. 3(c) and 3(d)]. It is interesting that we find a close correspondence between the correlation properties of measured and simulated fields: in both cases the correlation coefficient $\rho\left(\lambda_{1}, \lambda_{2}\right)$ decays over a finite wavelength shift, measurable in a half-width-half-maximum value of 0.22 (3) $\mathrm{nm}$ for the experiment and $0.26(3) \mathrm{nm}$ for the simulation. We can therefore exclude the different behavior of the short-living population of singularities to originate merely as a consequence of the finite spectral correlation of the random wave field.
We note that we ruled out the eventuality in which the short-living population of singularities is generated by experimental artifacts such as noise in the measurements, temperature fluctuations, or phase drifts. Independent measurements realized with different wavelength sweeps showed perfect consistency, demonstrating that variables that are not the wavelength shift cannot affect the final results [31]. Moreover, we introduced these as well as other possible measurement artifacts (i.e., perturbation from the near-field probe, frequency instabilities) in our models, in order to check if they could in any case lead to an enhanced population of faithful singularities at small $\Delta \lambda$; they did not show any of these changes.

Interestingly, we did find a model that reproduces the observed enhanced population. When a second family of eigenstates with a spectral width $\gamma^{\prime}$ different from the one of the original eigenstates is added to the decomposition of Eq. (2), two populations of singularities start to appear. Such an additional family of modes could be provided by resonances which, due to polarization or physical separation, would ideally remain orthogonal to the chaotic modes, but which get coupled to them in the real-life system. Figure 5 presents the persistence histogram for such a proposed model case, varying the ratio $\gamma / \gamma^{\prime}$. For some choices of $\gamma^{\prime}$ a biexponential behavior appears. Specifically, we observed that such choices satisfy $\Delta \simeq \gamma \gg \gamma^{\prime}$, in which cases an excess of faithful singularities living within the spectral correlation region of the field is again found [31].

To conclude, we studied the persistence and pairing statistics of phase singularities in optical random waves. For singularities with a persistence longer than the spectral correlation of the random field, we find perfect agreement between experiment, simulation, and theory. It is striking that, for singularities with a persistence that falls within the spectral correlation of the random field, we experimentally observe an excess of singularities compared to theoretical prediction, and these are more faithful than expected. With

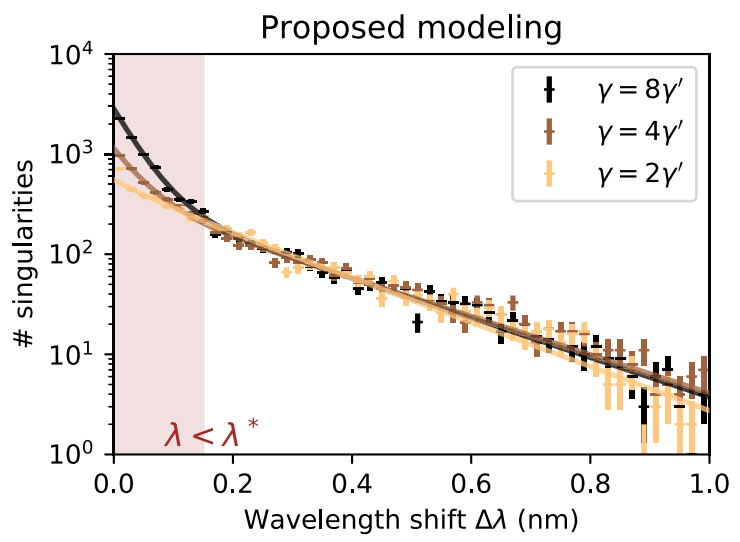

FIG. 5. Persistence histograms for singularities arising in numerically generated random fields in which two families of eigenstates with different spectral widths $\gamma$ and $\gamma^{\prime}$ coexist [31]. The lines are biexponential fits to the data points. 
this in view, we propose a mechanism based on the coexistence of different families of eigenstates, which could lead to a full explanation of our experimental observation.

The research data supporting this publication can be accessed at [32].

We thank Thomas Bauer for critical reading of the manuscript. This work is part of the research program of the Netherlands Organization for Scientific Research (NWO). The authors acknowledge funding from the European Research Council (ERC Advanced Grant No. 340438-CONSTANS). F. A. acknowledges support from the Marie Skłodowska-Curie individual fellowship BISTRO-LIGHT (Grant No. 748950). A. D. F. acknowledges support from EPSRC (Grants No. EP/L017008/1 and No. EP/M000869/1).

*1.kuipers@tudelft.nl

[1] M. W. Zwierlein, J. R. Abo-Shaeer, A. Schirotzek, C. H. Schunck, and W. Ketterle, Nature (London) 435, 1047 (2005).

[2] K. G. Lagoudakis, M. Wouters, M. Richard, A. Baas, I. Carusotto, R. André, L. S. Dang, and B. Deveaud-Plédran, Nat. Phys. 4, 706 (2008).

[3] O. M. Auslaender, L. Luan, E. W. Straver, J. E. Hoffman, N. C. Koshnick, E. Zeldov, D. A. Bonn, R. Liang, W. N. Hardy, and K. A. Moler, Nat. Phys. 5, 35 (2009).

[4] G. Roumpos, M. D. Fraser, A. Löffler, S. Höfling, A. Forchel, and Y. Yamamoto, Nat. Phys. 7, 129 (2011).

[5] F. Manni, K. Lagoudakis, T. H. Liew, R. André, V. Savona, and B. Deveaud, Nat. Commun. 3, 1309 (2012).

[6] L. Giomi, Phys. Rev. X 5, 031003 (2015).

[7] R. Barboza, U. Bortolozzo, M. G. Clerc, S. Residori, and E. Vidal-Henriquez, Adv. Opt. Photonics 7, 635 (2015).

[8] S. M. Barnett, Phys. Rev. Lett. 118, 114802 (2017).

[9] A. Souslov, B. C. van Zuiden, D. Bartolo, and V. Vitelli, Nat. Phys. (in press).

[10] J. M. Kosterlitz and D. J. Thouless, J. Phys. C 6, 1181 (1973).

[11] J. F. Nye and M. V. Berry, Proc. R. Soc. A 336, 165 (1974).

[12] M. V. Berry and M. R. Dennis, Proc. R. Soc. A 456, 2059 (2000).
[13] K. O’Holleran, M. R. Dennis, and M. J. Padgett, Phys. Rev. Lett. 102, 143902 (2009).

[14] R. Höhmann, U. Kuhl, H.-J. Stöckmann, J. D. Urbina, and M. R. Dennis, Phys. Rev. E 79, 016203 (2009).

[15] L. De Angelis, F. Alpeggiani, A. Di Falco, and L. Kuipers, Phys. Rev. Lett. 117, 093901 (2016).

[16] A. J. Taylor and M. R. Dennis, Nat. Commun. 7, 12346 (2016).

[17] D. Rozas, Z. S. Sacks, and G. A. Swartzlander, Phys. Rev. Lett. 79, 3399 (1997)

[18] K. O'Holleran, M. R. Dennis, F. Flossmann, and M. J. Padgett, Phys. Rev. Lett. 100, 053902 (2008).

[19] M. Dennis, R. King, B. Jack, K. O'Holleran, and M. Padgett, Nat. Phys. 6, 118 (2010).

[20] L. Giomi, M. J. Bowick, X. Ma, and M. C. Marchetti, Phys. Rev. Lett. 110, 228101 (2013).

[21] X. Cheng, Y. Lockerman, and A. Z. Genack, Opt. Lett. 39, 3348 (2014).

[22] N. Rotenberg, B. le Feber, T. D. Visser, and L. Kuipers, Optica 2, 540 (2015).

[23] M. V. Berry, Contemp. Phys. 56, 1 (2015).

[24] C. Liu, R. E. C. van der Wel, N. Rotenberg, L. Kuipers, T. F. Krauss, A. Di Falco, and A. Fratalocchi, Nat. Phys. 11, 358 (2015).

[25] M. L. M. Balistreri, J. P. Korterik, L. Kuipers, and N. F. van Hulst, Phys. Rev. Lett. 85, 294 (2000).

[26] M. Burresi, R. J. P. Engelen, A. Opheij, D. van Oosten, D. Mori, T. Baba, and L. Kuipers, Phys. Rev. Lett. 102, 033902 (2009).

[27] B. Le Feber, N. Rotenberg, D. M. Beggs, and L. Kuipers, Nat. Photonics 8, 43 (2014).

[28] H.-J. Stöckmann, Quantum Chaos: An Introduction (Cambridge University Press, Cambridge, England, 2006).

[29] G. Gbur, Optica 3, 222 (2016).

[30] M. V. Berry, J. Phys. A 10, 2083 (1977).

[31] See Supplemental Material at http://link.aps.org/ supplemental/10.1103/PhysRevLett.119.203903 for more details on nanofabrication, near-field microscopy, singularities tracking, experiment repeatability, numerical simulations and for one particular example from the proposed model with coexistence of two families of eigenstates.

[32] http://dx.doi.org/10.4121/uuid:1c716e9e-79e9-4db4-9d6bf95600ef9785. 